\title{
Asociación de la fiebre y el tratamiento antipirético con la progresión de la disfunción orgánica en sepsis: cohorte prospectiva
}

\section{Otoniel Toledo-Salinas, ${ }^{1 *}$ Luis A. Sánchez-Hurtado² y Juan Rodríguez-Silverio ${ }^{3}$}

${ }^{1}$ Instituto Mexicano del Seguro Social, Centro Médico Nacional La Raza, Hospital de Especialidades; ${ }^{2}$ nstituto Mexicano del Seguro Social, Centro Médico Nacional Siglo XXI, Hospital de Especialidades; ${ }^{3}$ Instituto Politécnico Nacional, Escuela Superior de Medicina, Sección de Estudios de Posgrado. Ciudad de México, México

\section{Resumen}

Introducción: No se conoce si la fiebre y el tratamiento antipirético se relacionan con progresión de la disfunción orgánica (PDO) en sepsis. Objetivo: Evaluar la asociación de la fiebre y el tratamiento antipirético con la PDO en sepsis. Métodos: Estudio de cohorte prospectiva de pacientes con sepsis. Se registró temperatura axilar máxima ( $T^{\circ}$ máx.), dosis total de fármacos antipiréticos y puntuación diaria de la escala SOFA. La PDO se definió como el incremento de SOFA $\geq 1$ punto. Se utilizó un modelo de regresión logística multivariado para evaluar la asociación estudiada. Resultados: Se incluyeron 305 pacientes: 163 mujeres (53.4\%) con puntuación SOFA de ocho puntos (6-11); 130 participantes (42.62 \%) presentaron $T^{\circ}$ máx. $\geq 38^{\circ} \mathrm{C}$ y $76(24.9 \%)$, PDO. La mortalidad en los pacientes con fiebre fue de $26.2 \%$ versus $20 \%$ sin fiebre $(p=0.21)$ y con PDO, de $73.7 \%$ versus $5.7 \%(p=0.01)$. La T $T^{\circ}$ máx. $\geq 39^{\circ} \mathrm{C}$ tuvo $R M=4.96$ (IC $\left.95 \%=1.97-12.47, p=0.01\right)$ y el uso de antipiréticos, $R M=1.04$ (IC $95 \%=0.58-1.86, p=0.88$ ). Conclusiones: $L a T^{\circ}$ máx. axilar $\geq 39^{\circ} \mathrm{C}$ es un factor de riesgo para PDO en sepsis. El uso de antipiréticos no se asoció a PDO.

PALABRAS CLAVE: Fiebre. Antipirético. Sepsis. Disfunción orgánica.

\section{Association of fever and antipyretic treatment with progressive organ dysfunction in sepsis: Prospective cohort}

\begin{abstract}
Introduction: Whether fever and antipyretic treatment are related to progression of organ dysfunction (POD) in sepsis is not known. Objective: To evaluate the association of fever and antipyretic treatment with POD in sepsis. Methods: Prospective cohort study of patients with sepsis. Maximum axillary temperature ( $T^{\circ} \mathrm{Max}$ ), antipyretic drugs total dose and daily SOFA score were recorded. POD was defined as an increase $\geq 1$ point on the SOFA score. A multivariate logistic regression model was used to evaluate the studied association. Results: 305 patients were included: 163 were women (53.4\%), with a SOFA score of 8 points (6-11); 130 participants (42.62 \%) had $T^{\circ} \mathrm{Max} \geq 38{ }^{\circ} \mathrm{C}$, and 76 (24.9\%), POD. Mortality in patients with fever was $26.2 \%$ vs. $20 \%(p=0.21)$, and with $P O D, 73.7 \%$ vs. $5.7 \%(p=0.01)$. $T^{\circ} \mathrm{Max} \geq 39{ }^{\circ} \mathrm{C}$ had an OR of $4.96(95 \% \mathrm{Cl}=1.97$ 12.47, $p=0.01$ ); and the use of antipyretics, an OR of 1.04 (95\% Cl: 0.58-1.86, $p=0.88$ ). Conclusions: An axillary $T^{\circ}$ Max $\geq 39^{\circ} \mathrm{C}$ is a risk factor for $P O D$ in sepsis. The use of antipyretics was not associated with POD.
\end{abstract}

KEY WORDS: Fever. Antipyretic. Sepsis. Organ dysfunction.

Correspondencia:

*Otoniel Toledo-Salinas

E-mail: otoniel_toledo@live.com.mx

0016-3813/๑ 2020 Academia Nacional de Medicina de México, A.C. Publicado por Permanyer. Este es un artículo open access bajo la licencia CC BY-NC-ND (http://creativecommons.org/licenses/by-nc-nd/4.0/).
Fecha de recepción: 22-10-2020

Fecha de aceptación: 10-12-2020

DOI: $10.24875 / G M M .20000756$
Gac Med Mex. 2021:157:391-397

Disponible en PubMed www.gacetamedicademexico.com
tículo open access bajo la licencia 


\section{Introducción}

La sepsis es un síndrome de disfunción orgánica ocasionado por la respuesta desregulada del hospedero hacia una infección. ${ }^{1}$ Al menos $6 \%$ de los pacientes hospitalizados presenta sepsis y la mortalidad varía de 26 a $58 \%{ }^{2}{ }^{2}$

La disfunción orgánica asociada a sepsis no es un evento único sino un proceso de daño orgánico continuo originado por diferentes mecanismos que interactúan mutuamente. ${ }^{3}$ La evaluación objetiva del grado de disfunción orgánica en el paciente con sepsis se realiza diariamente mediante la escala SOFA (Sequential Organic Failure Assesment). ${ }^{4}$ El incremento de la puntuación SOFA, es decir, la progresión de la disfunción orgánica (PDO), se asocia a incremento en la mortalidad. ${ }^{5}$

La fiebre se define como la presencia de una temperatura corporal axilar $\geq 38.3^{\circ} \mathrm{C}$ en el paciente críticamente enfermo. ${ }^{6}$ Se presenta en $50 \%$ de todos los ingresos a la unidad de cuidados intensivos ( $\mathrm{UCl}$ ) y hasta $44 \%$ de los pacientes con sepsis tiene fiebre al momento del diagnóstico. ${ }^{7}$ La campaña de Sobreviviendo a la Sepsis no hace ninguna recomendación acerca de su tratamiento ni de la meta de temperatura. $^{8}$

La fiebre coexiste en equilibrio con la respuesta inflamatoria como adyuvante para las funciones inmunológicas de erradicación de la infección. ${ }^{9}$ El tratamiento farmacológico estricto de la fiebre ocasiona reducción en el consumo de oxígeno y mejoría en el estado de choque circulatorio; ${ }^{10}$ sin embargo, podría reducir la habilidad inmunológica y tener efectos adversos en pacientes que presentan algún grado de disfunción orgánica. ${ }^{11,12}$ Existen dos posturas respecto al tratamiento de la fiebre en el paciente con sepsis: "suprimir" y "dejar andar"; la primera postura continúa siendo el dogma en vez de la práctica basada en la evidencia. ${ }^{13}$ Para dar respuesta a la incertidumbre clínica hemos realizado un estudio con el objetivo principal de evaluar la asociación de la fiebre y del tratamiento antipirético con la PDO en los pacientes con sepsis.

\section{Métodos}

Se realizó un estudio observacional, longitudinal y prospectivo de junio de 2018 a marzo de 2020 en una $\mathrm{UCI}$ de tercer nivel con pacientes $\geq 16$ años diagnosticados con sepsis (Sepsis-3), ${ }^{1}$ y que disponían de consentimiento informado. Se excluyeron los pacientes con choque séptico refractario (norepinefrina $>0.5 \mu \mathrm{g} / \mathrm{kg} /$ minuto, tensión arterial media $<65 \mathrm{~mm} \mathrm{Hg}$ ), en estado de posreanimación cardiopulmonar y pacientes neurocríticos; se eliminaron quienes murieron en las primeras 24 horas de la estancia en la UCI (Figura 1). Se midió la temperatura axilar de forma horaria con termómetro de mercurio. Los pacientes se agruparon conforme la temperatura axilar máxima $\left(T^{\circ}\right.$ máx.) durante su estancia en la UCl: $36-37.9{ }^{\circ} \mathrm{C}$, $38-38.9^{\circ} \mathrm{C}$ y $\geq 39^{\circ} \mathrm{C}$. Diariamente se registró la puntuación SOFA ${ }^{4}$ y el tipo de antipirético recibido con su posología. La fiebre se definió como $\mathrm{T}^{\circ}$ máx. $\geq 38.3^{\circ} \mathrm{C}$ o $\mathrm{T}^{\circ}$ máx. $\geq 38^{\circ} \mathrm{C}$ en al menos dos mediciones. ${ }^{\circ} \mathrm{En}$ tanto, la PDO se precisó como un incremento de SOFA $\geq 1$ punto al egreso de la unidad.

Se realizó el cálculo de la muestra con un nivel alfa de 0.05 y poder estadístico de $80 \%$ para detectar una razón de probabilidades (RM, razón de momios) $\geq 2$. Para determinar el tipo de distribución de las variables, se realizó la prueba de normalidad de Kolmogorov-Smirnov. Las variables cuantitativas fueron analizadas con las pruebas $t$ de Student $y$ prueba $U$ de Mann-Whitney de acuerdo con su tipo de distribución. Las variables cualitativas fueron analizadas con $\chi^{2}$. Se realizó análisis de regresión logística univariado y multivariado para la $\mathrm{T}^{\circ}$ máx. $\geq 39{ }^{\circ} \mathrm{C}$ y el tratamiento antipirético. Se realizó un análisis de supervivencia para los grupos de $\mathrm{T}^{\circ}$ máx. mediante la curva de Kaplan-Meier y la regresión de Cox. En todos los casos, un valor de $p<0.05$ fue considerado estadísticamente significativo.

El análisis de los datos se realizó utilizando el programa estadístico SPSS versión 25.0.

\section{Resultados}

Se revisaron los datos de 1255 pacientes que ingresaron de forma consecutiva a la $\mathrm{UCl}$, de los cuales 305 contaron con los criterios de inclusión para su análisis (Figura 1).

La mediana de edad fue de 54 años (38-64) y la de $\mathrm{SOFA}^{4}$ de ocho puntos (6-11). Las infecciones más frecuentes fueron de origen abdominal $(42 \%)$ y el choque séptico estuvo presente en $75.7 \%$ de los casos; $42.6 \%$ de los pacientes con sepsis presentó fiebre durante su estancia en la UCl. Los pacientes con fiebre requirieron mayor número de días de ventilación mecánica: ocho (4-13) versus cuatro (2-8), $p=0.01$; más días de uso de vasopresores: cuatro 


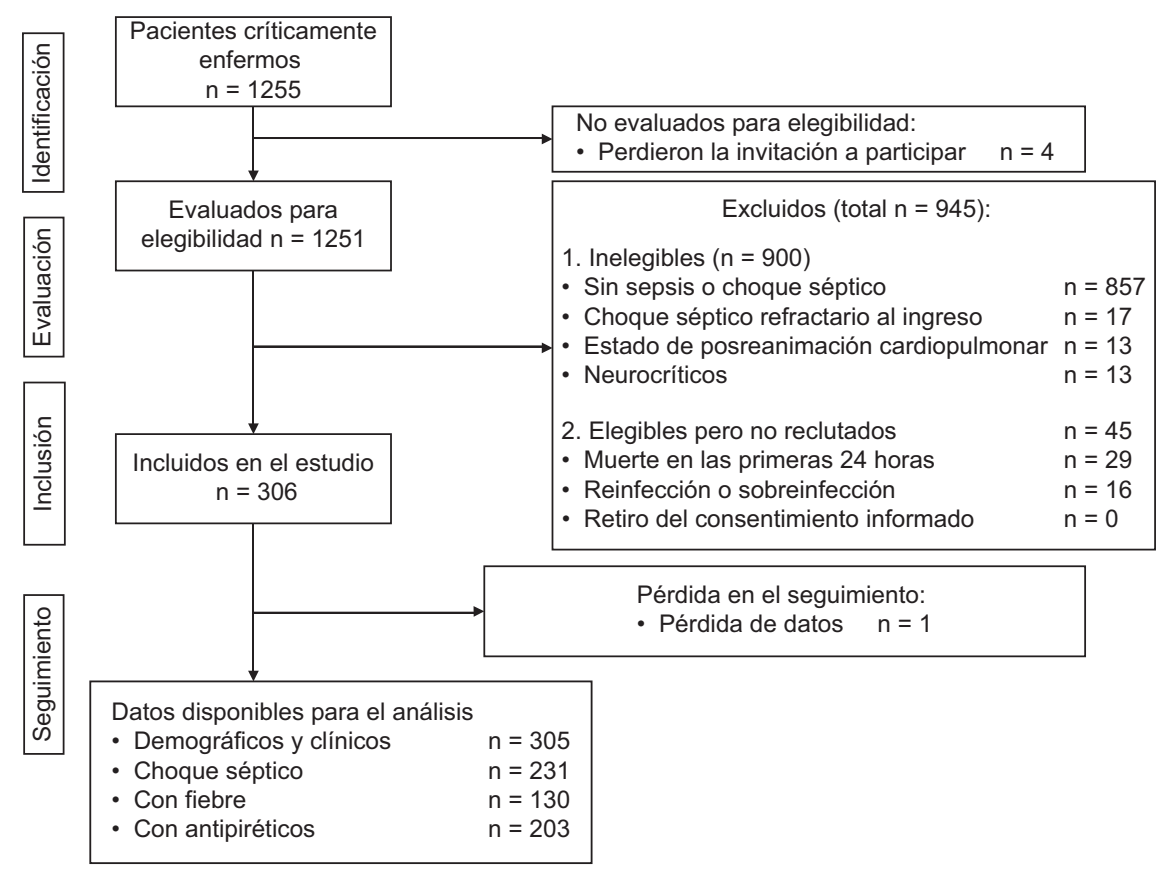

Figura 1. Diagrama de flujo que describe las etapas de la investigación.

(1-7) versus tres (1-5), $p=0.01$; más días de estancia en la UCl: 10 (5-14) versus seis (3-10), $p=0.01$; $y$ mayor porcentaje de fracaso en el retiro de la ventilación mecánica: $33.1 \%$ versus $16.6 \%, p=0.01$. No se observó diferencia estadísticamente significativa en la PDO: $30 \%$ versus $21.1 \%, p=0.07$; ni en la mortalidad: $26.2 \%$ versus $20 \%, p=0.21$ (Tabla 1).

Los tipos de disfunción orgánica más frecuentes en los pacientes con y sin PDO fueron la disfunción respiratoria: $98.7 \%$ versus $98.7 \%, p=0.99$; la disfunción hemodinámica: $76.3 \%$ versus $72.5 \%, p=0.51$; y la disfunción renal: $57.9 \%$ versus $51.1 \%, p=0.30$.

La mediana de $\mathrm{T}^{\circ}$ máx. de los pacientes con y sin PDO fue de $38^{\circ} \mathrm{C}(36.8-38.5)$ versus $37.5^{\circ} \mathrm{C}$ (37-38.3), $p=0.31$; el porcentaje de uso de antipiréticos: $51.3 \%$ versus $45 \%, p=0.33$ (paracetamol enteral: $36.8 \%$ versus $31 \%, p=0.34$; metamizol intravenoso: $43.4 \%$ versus $31 \%, p=0.04)$. La mediana de la dosis de paracetamol enteral fue de $1 \mathrm{~g}(0-4)$ versus $0 \mathrm{~g}(0-1)$, $p=0.01 ; y$ de metamizol intravenoso: $3 g(2-11)$ versus $0 \mathrm{~g}(0-1), \mathrm{p}=0.01$.

Los pacientes con PDO mostraron mayor cantidad de días de ventilación mecánica: ocho (4-12) versus 5 (2-10), $p=0.01$; días con vasopresores: seis (4-11) versus dos (1-5), $\mathrm{p}=0.01 ; \mathrm{y}$ mortalidad: $73.7 \%$ versus $5.7 \%, p=0.01$. El porcentaje de PDO en el grupo con $\mathrm{T}^{\circ}$ máx. $\geq 39{ }^{\circ} \mathrm{C}(8.9 \%)$ y en el grupo de $\mathrm{T}^{\circ}$ máx. de $36-38.9{ }^{\circ} \mathrm{C}(91.1 \%)$ fue de $51.9 \%$ versus $22.3 \%$,

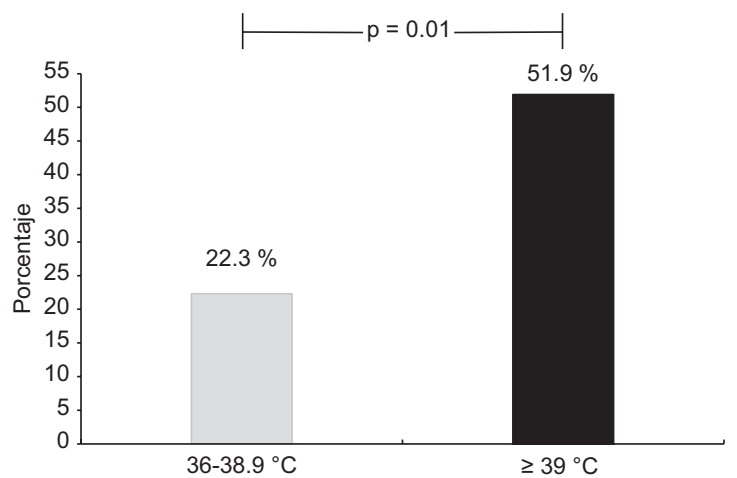

Figura 2. Porcentaje de progresión de la disfunción orgánica al egreso de la $\mathrm{UCl}$ en los pacientes con $\mathrm{T}^{\circ}$ máx. de $36-38.9{ }^{\circ} \mathrm{C}$ y $\geq 39^{\circ} \mathrm{C} . T^{\circ}$ máx. = temperatura axilar máxima durante la estancia en la $\mathrm{UCl}, \mathrm{UCl}=$ Unidad de Cuidados Intensivos.

$p=0.01$ (Figura 2); en tanto, la mortalidad fue de $37 \%$ versus $21.2 \%, p=0.06$. La mediana de días de ventilación mecánica para el grupo con $\mathrm{T}^{\circ}$ máx. $\geq 39^{\circ} \mathrm{C}$ fue de 11 (3-14) versus cinco (3-11), $p=0.18$; la mediana de días de vasopresores fue de cinco (3-10) versus tres $(1-6), p=0.03$; y la mediana de días de estancia en $\mathrm{UCl}$ fue de 12 (4-14) versus siete (4-12), $p=0.13$.

En el análisis de regresión logística univariado, la fiebre tuvo $\mathrm{RM}=1.59$ (IC $95 \%=0.94-2.69$, $p=0.07$ ), mientras que el grupo con $T^{\circ}$ máx. $\geq 39^{\circ} \mathrm{C}$ presentó RM = 3.75 (IC $95 \%$ : 1.67-8.41, p = 0.01). La 
Tabla 1. Características demográficas y clínicas de los pacientes con sepsis al ingreso a la UCl y comparación de los pacientes con y sin fiebre

\begin{tabular}{|c|c|c|c|c|c|c|c|}
\hline \multirow[t]{2}{*}{ Caracteristica } & \multicolumn{2}{|c|}{$\begin{array}{l}\text { Muestra } \\
(n=305)\end{array}$} & \multicolumn{2}{|c|}{$\begin{array}{l}\text { Con fiebre } \\
(n=130)\end{array}$} & \multicolumn{2}{|c|}{$\begin{array}{l}\text { Sin fiebre } \\
(n=175)\end{array}$} & \multirow[t]{2}{*}{$p$} \\
\hline & Mediana & RIC & Mediana & RIC & Mediana & RIC & \\
\hline Edad, años & 54 & $38-64$ & 51 & $36-63$ & 57 & $41-66$ & 0.08 \\
\hline Índice de Charlson, puntuación & 1 & $0-2$ & 1 & $0-2$ & 2 & $0-2$ & 0.19 \\
\hline APACHE II, puntuación & 15 & $12-19$ & 15 & $12-19$ & 16 & $11-19$ & 0.66 \\
\hline SOFA, puntuación & 8 & $6-11$ & 9 & $6-11$ & 8 & $5-11$ & 0.88 \\
\hline Ventilación mecánica, días & 5 & $3-11$ & 8 & $4-13$ & 4 & $2-8$ & 0.01 \\
\hline Vasopresores, días & 3 & $1-6$ & 4 & $1-7$ & 3 & $1-5$ & 0.01 \\
\hline \multirow{2}{*}{ Estancia en UCI, días } & 7 & 4-12 & 10 & $5-14$ & 6 & $3-10$ & 0.01 \\
\hline & $\mathrm{n}$ & $\%$ & $\mathrm{n}$ & $\%$ & $\mathrm{n}$ & $\%$ & \\
\hline Sexo, mujeres & 163 & 53.4 & 58 & 44.6 & 105 & 60 & 0.01 \\
\hline $\begin{array}{l}\text { Comorbilidades } \\
\text { Enfermedad renal crónica } \\
\text { Insuficiencia cardiaca crónica } \\
\text { Enfermedad oncológica } \\
\text { Enfermedad pulmonar obstructiva } \\
\text { Insuficiencia hepática crónica }\end{array}$ & $\begin{array}{l}39 \\
27 \\
26 \\
19 \\
14\end{array}$ & $\begin{array}{l}12.8 \\
8.9 \\
8.5 \\
6.2 \\
4.6\end{array}$ & $\begin{array}{l}14 \\
9 \\
7 \\
6 \\
7\end{array}$ & $\begin{array}{l}10.8 \\
6.9 \\
5.4 \\
4.6 \\
5.4\end{array}$ & $\begin{array}{l}25 \\
18 \\
19 \\
13 \\
7\end{array}$ & $\begin{array}{c}14.3 \\
10.3 \\
10.9 \\
7.4 \\
4\end{array}$ & $\begin{array}{l}0.36 \\
0.30 \\
0.09 \\
0.31 \\
0.56\end{array}$ \\
\hline Inmunosupresores & 44 & 14.4 & 17 & 13.1 & 27 & 15.4 & 0.56 \\
\hline Paciente quirúrgico & 180 & 59 & 80 & 61.5 & 100 & 57.1 & 0.44 \\
\hline Infección adquirida en comunidad & 169 & 55.4 & 67 & 51.5 & 102 & 58.3 & 0.62 \\
\hline $\begin{array}{l}\text { Sitio de infección } \\
\text { Abdominal } \\
\text { Pulmonar } \\
\text { Tejidos blandos } \\
\text { Urinario }\end{array}$ & $\begin{array}{c}128 \\
106 \\
34 \\
31\end{array}$ & $\begin{array}{c}42 \\
34.8 \\
11.1 \\
10.2\end{array}$ & $\begin{array}{l}52 \\
48 \\
17 \\
11\end{array}$ & $\begin{array}{c}40 \\
36.9 \\
13.1 \\
8.5\end{array}$ & $\begin{array}{l}76 \\
58 \\
17 \\
20\end{array}$ & $\begin{array}{c}43.4 \\
33.1 \\
9.7 \\
11.4\end{array}$ & $\begin{array}{l}0.54 \\
0.49 \\
0.35 \\
0.39\end{array}$ \\
\hline Cultivo positivo & 185 & 60.7 & 81 & 62.3 & 104 & 59.4 & 0.61 \\
\hline $\begin{array}{l}\text { Tipo de microorganismo } \\
\text { Gramnegativo } \\
\text { Grampositivo }\end{array}$ & $\begin{array}{c}127 \\
34\end{array}$ & $\begin{array}{l}68.6 \\
18.3\end{array}$ & $\begin{array}{c}59 \\
9\end{array}$ & $\begin{array}{l}72.8 \\
11.1\end{array}$ & $\begin{array}{l}68 \\
25\end{array}$ & $\begin{array}{c}65.3 \\
24\end{array}$ & $\begin{array}{l}0.30 \\
0.02\end{array}$ \\
\hline Choque séptico & 231 & 75.7 & 132 & 75.4 & 99 & 76.2 & 0.88 \\
\hline Paracetamol & 99 & 32.5 & 74 & 56.9 & 25 & 14.3 & 0.01 \\
\hline Metamizol & 104 & 34.1 & 98 & 75.4 & 6 & 3.4 & 0.01 \\
\hline Fracaso en el retiro de la ventilación & 72 & 23.6 & 43 & 33.1 & 29 & 16.6 & 0.01 \\
\hline Progresión de la disfunción orgánica & 76 & 24.9 & 39 & 30 & 37 & 21.1 & 0.07 \\
\hline Muerte & 69 & 22.6 & 34 & 26.2 & 35 & 20 & 0.21 \\
\hline
\end{tabular}

APACHE II = Acute Physiology and Chronic Health Evaluation, RIC = rango intercuartilar, SOFA = Sequential Organic Failure Assessment, UCI = Unidad de Cuidados Intensivos.

administración de antipiréticos tuvo RM $=1.28$ (IC $95 \%=0.76-2.16, p=0.33$ ): el paracetamol enteral mostró $\mathrm{RM}=1.29$ (IC $95 \%=0.75-2.23$, $\mathrm{p}=0.34$ ) y el metamizol intravenoso, $\mathrm{RM}=1.71$ (IC $95 \%=1.01-2.91, p=0.04)$, como puede observarse en la Tabla 2.
En la regresión logística multivariada ajustada respecto a la edad, puntuación APACHE II4 $>15$ puntos, infección nosocomial, choque séptico, tratamiento antibiótico previo al ingreso a $\mathrm{UCl}$ y uso de antipiréticos, la $\mathrm{T}^{\circ}$ máx. $\geq 39{ }^{\circ} \mathrm{C}$ tuvo $\mathrm{RM}=4.95$ (IC $95 \%=1.37-12.47, p=0.01$ ) y el uso de 
Tabla 2. Análisis univariado de variables asociadas a la progresión de la disfunción orgánica al egreso de la $\mathrm{UCl}$ en pacientes sépticos

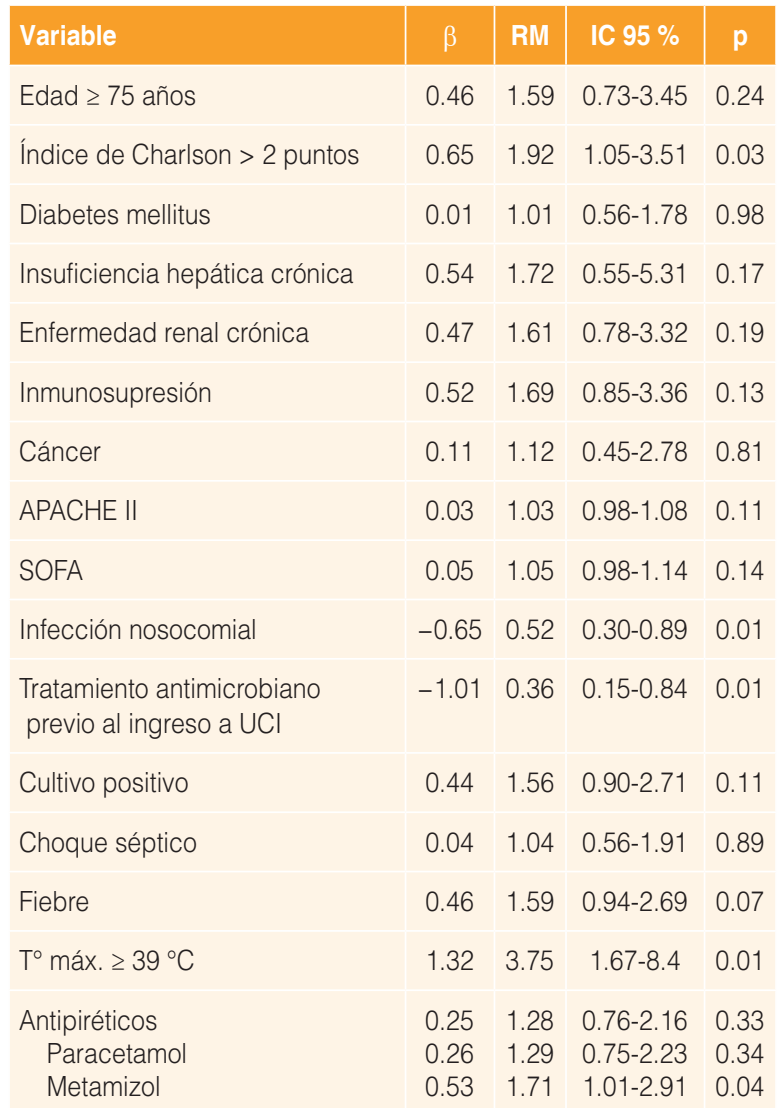

antipiréticos $\mathrm{RM}=1.04$ (IC $95 \%=0.58-1.86, \mathrm{p}=0.88)$, como se describe en la Tabla 3.

La mediana de días libres de PDO fue de 19 (IC $95 \%=16-21.99$ ) y la prueba de log-rank entre los diferentes grupos de $T^{\circ}$ máx. no mostró significación estadística (Figura 3).

\section{Discusión}

\section{Resultados clave}

Identificamos que la fiebre $\geq 39{ }^{\circ} \mathrm{C}$ constituye un factor de riesgo para la PDO, mientras que el uso de antipiréticos no se comportó de la misma forma.

La proporción de pacientes con $\mathrm{T}^{\circ}$ máx. $\geq 38{ }^{\circ} \mathrm{C}$ fue de $42.62 \%$, similar a la reportada por Laupland et al. ${ }^{15}(44 \%)$, mientras que Young et $a l .^{16}$ identificaron fiebre en $20 \%$ de los pacientes con sepsis severa, lo cual difiere con nuestros resultados debido a que la temperatura registrada fue el promedio diario de cuatro mediciones; no obstante, la mortalidad referida fue
Tabla 3. Análisis de regresión logística multivariado de variables asociadas a la progresión de la disfunción orgánica en pacientes sépticos

\begin{tabular}{|l|c|c|c|c|}
\hline Variable & $\beta$ & RM & IC 95 \% & $p$ \\
\hline Edad $\geq 75$ años & 0.58 & 1.79 & $0.77-4.11$ & 0.17 \\
\hline \begin{tabular}{l} 
Índice de Charlson > 2 puntos \\
\hline Apache II
\end{tabular} & 0.56 & 1.75 & $0.91-3.35$ & 0.08 \\
\hline $\begin{array}{l}\text { Infección nosocomial } \\
\text { Choque séptico }\end{array}$ & 0.04 & 1.04 & $0.99-1.10$ & 0.07 \\
\hline$T^{\circ}$ máx. $\geq 39{ }^{\circ} \mathrm{C}$ & -0.51 & 0.59 & $0.33-1.07$ & 0.59 \\
\hline $\begin{array}{l}\text { Tratamiento antibiótico previo } \\
\text { al ingreso a UCl }\end{array}$ & -0.33 & 0.71 & $0.36-1.39$ & 0.32 \\
\hline \begin{tabular}{l} 
Antipiréticos \\
\hline
\end{tabular} & -1.07 & 4.96 & $1.97-12.47$ & 0.01 \\
\hline
\end{tabular}

$\chi^{2}=30.54$, prueba ómnibus: $p=0.001, R^{2}$ de Nagelkerke $=0.14$ Prueba de Hosmer-Lemeshow: $p=0.18$, porcentaje global $=74.4 \%$,

sensibilidad $=45 \%$, especificidad $=76 \%$, valor predictivo positivo $=14 \%$, valor predictivo negativo $=94 \%$, área bajo la curva ROC $=0.71($ IC $95 \%=0.65-0.77, p=0.01)$. APACHE $=$ Acute Physiologic and Chronic Health Evaluation, $T^{\circ}$ máx. = temperatura máxima durante la estancia en la $\mathrm{UCl}, \mathrm{UCl}=$ Unidad de Cuidados Intensivos.

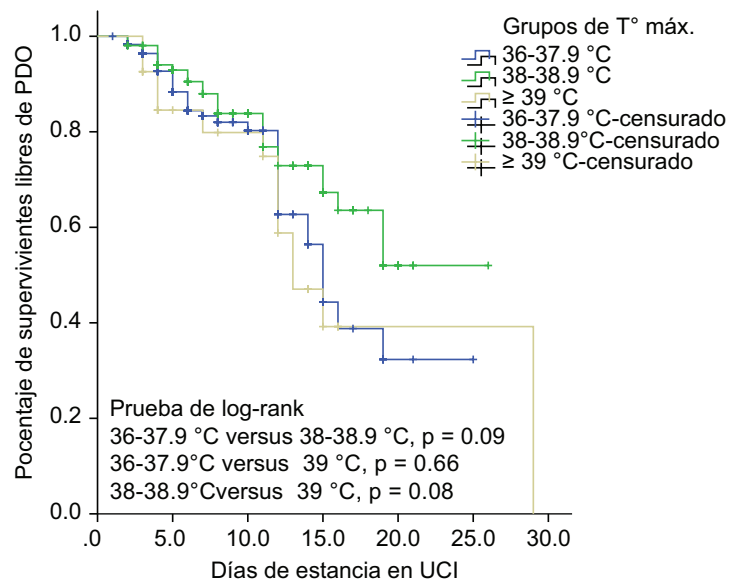

Figura 3. Porcentaje de pacientes supervivientes libres de PDO por grupo de $T^{\circ}$ máx. $P D O=$ progresión de la disfunción orgánica al egreso de la $\mathrm{UCl}, \mathrm{T}^{\circ}$ máx. = temperatura máxima durante la estancia en la UCl. UCl = Unidad de Cuidados Intensivos.

de $16 \%$, similar a la encontrada en nuestro estudio (19.3\%).

Hasta donde sabemos, no existe un análisis previo en pacientes con sepsis que tenga como objetivo principal evaluar la asociación de la fiebre con la PDO (medida mediante la escala SOFA ${ }^{4}$ ) como desenlace centrado en el paciente que pudiera tener mayor importancia clínica que la mortalidad. ${ }^{17}$ Ferreira et $a l . .^{5}$ reportaron que el incremento en la puntuación de disfunción orgánica 
se relaciona con un peor desenlace, similar a lo encontrado en nuestro estudio. Por su parte, Kushimoto et al. ${ }^{18}$ no hallaron diferencia en la mortalidad entre los grupos de pacientes con sepsis severa con temperatura $\leq 36.5{ }^{\circ} \mathrm{C}$ o $>36.5{ }^{\circ} \mathrm{C}(21.5 \%$ versus $13.4 \%$, $\mathrm{p}=0.09$ ). A distinta temperatura (máxima $\geq 38^{\circ} \mathrm{C}$ ) tampoco registramos diferencia en la mortalidad ni en la PDO, en comparación con el grupo eutérmico.

\section{Fiebre y antipiréticos como factores de riesgo para la PDO}

Laupland et al. ${ }^{15}$ reportaron que los pacientes con temperatura $\geq 39.5^{\circ} \mathrm{C}$ tuvieron $\mathrm{RM}=1.91$ (1.36-2.7), similar a la encontrada en nuestro estudio. Por otra parte, Lee et al., ${ }^{7}$ con criterios de exclusión similares a los de nuestra investigación, reportaron que con la definición previa a Sepsis- $3,{ }^{1}$ la $\mathrm{T}^{\circ}$ máx. de $38.5^{\circ} \mathrm{C}$ a $39.4{ }^{\circ} \mathrm{C}$ no fue un factor de riesgo para mortalidad $(\mathrm{RM}=0.52,0.24-1.1$, $p=0.09$ ), lo cual contrasta con nuestro hallazgo cuando realizamos la comparación con la $\mathrm{T}^{\circ}$ máx. $\geq 39^{\circ} \mathrm{C}$.

Fujishima et al. ${ }^{19}$ y Paoli et al. ${ }^{20}$ señalaron que la disfunción respiratoria y la hemodinámica se presentaron en 40.2 y $29.7 \%$ de los pacientes con sepsis, respectivamente; en nuestro estudio, la disfunción respiratoria y la disfunción hemodinámica estuvieron presentes en mayor medida. El grupo con PDO no mostró diferencia en la proporción que recibió paracetamol en comparación con los pacientes sin PDO (paracetamol enteral $36.8 \%$ versus $31 \%, p=0.34$ ); sin embargo, una mayor proporción del grupo con PDO recibió metamizol intravenoso ( $43.4 \%$ versus $31 \%, p=0.04)$. A pesar de ello, la mediana de la dosis total de cada fármaco no alcanzó la posología que, conforme la literatura, se asocia con mayor riesgo de efectos adversos:11,12 paracetamol enteral, $0 \mathrm{~g}$, rango intercuartilar $(\mathrm{RIC})=0-1$ versus $0 \mathrm{~g}$, $\mathrm{RIC}=0-3, \mathrm{p}=0.31$; metamizol intravenoso, $0 \mathrm{~g}, \mathrm{RIC}=$ $0-1$ versus $0 \mathrm{~g}, \mathrm{RIC} 0-3, p=0.06$. Por ello, los autores atribuimos esta diferencia a la dificultad en el control de la temperatura o a la cancelación de la vía enteral (mayor gravedad clínica).

Young et al. ${ }^{21}$ reportaron que en pacientes con sospecha de sepsis severa, el paracetamol no se asoció con mortalidad a 28 días en comparación con el grupo placebo: $\mathrm{RR}=1.00$ (IC $95 \%=0.67-1.5, \mathrm{p}=0.94$ ). Drewry et al. ${ }^{22}$ mostraron que el tratamiento antipirético con paracetamol y antiinflamatorios no esteroideos no se asoció con disminución en la mortalidad a 28 días: resultados experimentales, $\mathrm{I}^{2}=0 \%, \mathrm{RM}=0.93$ (IC $95 \%=0.79-1.09$ ) y resultados observacionales, $I^{2}$
$=76.1 \%, \mathrm{RM}=0.9$ (IC $95 \%=0.54-1.51)$. Lo anterior es similar a lo encontrado en nuestro estudio.

\section{Supervivencia de los grupos de $T^{\circ}$ máx.}

Lee et al. ${ }^{7}$ y Kushimoto et al. ${ }^{18}$ reportaron que no encontraron diferencia en la mortalidad a 28 días en los grupos de temperatura: $\geq 39.5^{\circ} \mathrm{C}, 37.5-38.4{ }^{\circ} \mathrm{C}$ y 38.5 $39.4^{\circ} \mathrm{C}$ versus el grupo de referencia: $36.5-37.4^{\circ} \mathrm{C}$. Con una agrupación diferente de la $\mathrm{T}^{\circ}$ máx. tampoco encontramos diferencia estadísticamente significativa en la supervivencia libre de PDO; sin embargo, cabe resaltar que la mediana de temperatura de los pacientes que presentaron PDO (mortalidad de $73.7 \%$ versus $5.7 \%$, $\mathrm{p}=0.01)$ fue de $38{ }^{\circ} \mathrm{C}\left(36.8-38.5^{\circ} \mathrm{C}\right)$ versus $37.5^{\circ} \mathrm{C}$ $\left(37-38.3^{\circ} \mathrm{C}\right), p=0.31$, por lo que pensamos que la diferencia en la supervivencia solo es posible encontrarla en el grupo con temperatura $\geq 39^{\circ} \mathrm{C}$.

\section{Limitaciones}

Primera: nuestro diseño fue observacional sin un método estandarizado para el tratamiento antipirético, por lo que los hallazgos solo pueden mostrar asociación y no causalidad de la PDO.

Segunda: se debieron excluir los pacientes $\geq 75$ años debido a los cambios en el patrón de la temperatura corporal, por lo que se sugieren nuevos estudios en este grupo etario. ${ }^{23}$

Tercera: se incluyeron en el análisis únicamente los dos fármacos antipiréticos más usados; sin embargo, todos los pacientes recibieron algún tipo de antimicrobiano, por lo que creemos que el efecto del tratamiento en la PDO pudiera haberse diluido en la muestra. Por otro lado, el uso de otros antiinflamatorios no esteroideos fue infrecuente (se prefirió el uso de opioides), por lo que su efecto modificador del desenlace fue pequeño. ${ }^{24}$

Cuarta: la Sociedad Americana de Enfermedades Infecciosas no recomienda el uso de termómetros axilares, sin embargo, el uso de este método es frecuente debido a su naturaleza no invasiva, accesibilidad, facilidad de uso y bajo costo en comparación con los métodos invasivos que, además, representan un factor de riesgo para el desarrollo de infecciones nosocomiales. ${ }^{6}$

Quinta: no se evaluó el papel del enfriamiento externo no farmacológico activo debido a que la $\mathrm{UCl}$ en donde se realizó la investigación no dispone de 
los dispositivos necesarios para su aplicación y monitorización.

\section{Implicaciones prácticas}

Sugerimos que en pacientes con sepsis y con características similares a las de nuestra población se realice el manejo permisivo de la $\mathrm{T}^{\circ}$ máx. de 38-38.5 ${ }^{\circ} \mathrm{C}$, mientras que el manejo antipirético farmacológico intensivo farmacológico solo se inicie en los enfermos con $\mathrm{T}^{\circ}$ máx. $>38.5^{\circ} \mathrm{C}$.

\section{Conclusiones}

La $\mathrm{T}^{\circ}$ máx. $\geq 39{ }^{\circ} \mathrm{C}$ durante la estancia en la $\mathrm{UCl}$ es un factor de riesgo para la PDO en los pacientes con sepsis. Por su parte, el uso de fármacos antipiréticos en los pacientes con sepsis no es un factor de riesgo para la PDO.

\section{Agradecimientos}

Al Centro de Adiestramiento en Investigación Clínica del Instituto Mexicano del Seguro Social (CAIC-IMSS).

\section{Conflicto de intereses}

Los autores declaran no tener conflicto de intereses alguno.

\section{Financiamiento}

La presente investigación no recibió ninguna beca específica de agencias de los sectores públicos, comercial o sin ánimos de lucro.

\section{Responsabilidades éticas}

Protección de personas y animales. Los autores declaran que los procedimientos se realizaron conforme las normas éticas del comité de experimentación humana responsable y de acuerdo con la Asociación Médica Mundial y la Declaración de Helsinki.

Confidencialidad de los datos. Los autores declaran que siguieron los protocolos de su centro de trabajo sobre la publicación de datos de pacientes.

Derecho a la privacidad y consentimiento informado. Los autores obtuvieron el consentimiento informado de los pacientes o sujetos referidos en el artículo, documento que obra en poder del autor de correspondencia.

\section{Bibliografía}

1. Singer M, Deutschman CS, Seymour CW, Shankar-Hari M, Annane D, Bauer M, et al. The Third International Consensus Definitions for Sepsis and Septic Shock (Sepsis-3). JAMA. 2016;315:801-810.

2. Rhee C, Dantes R, Epstein L, Murphy D, Seymour C, Iwashyna T, et al. Incidence and trends of sepsis in US hospitals using clinical vs claims data. 2009-2014. JAMA. 2017;318:1241-1249.

3. Lelubre C, Vincent, J-L. Mechanisms and treatment of organ failure in sepsis. Nat Rev Nephrol. 2018;14:417-427.

4. Vincent JL, Moreno R, Takala J, Willatts S, De Mendonça A, Bruining H, et al. The SOFA (Sepsis-related Organ Failure Assessment) score to describe organ dysfunction/failure. On behalf of the Working Group on Sepsis-Related Problems of the European Society of Intensive Care Medicine Intensive Care Medicine. Intensive Care Med. 1996;22: 707-710.

5. Ferreira FL, Bota DP, Bross A, Mélot C, Vincent JL. Serial evaluation of the SOFA score to predict outcome in critically ill patients. JAMA 2001;286:1754-1758.

6. O'Grady NP, Barie PS, Bartlett JG, Bleck T, Carroll K, Kalil AC, et al. Guidelines for evaluation of new fever in critically ill adult patients: 2008 update from the American College of Critical Care Medicine and the Infectious Diseases Society of America. Crit Care Med. 2008;36: 1330-1349.

7. Lee B, Inui D, Suh G, Kim J, Kwon J, Park J, et al. Association of body temperature and antipyretic treatments with mortality of critically ill patients with and without sepsis: multi-centered prospective observational study. Critical Care. 2012;16:R33.

8. Rhodes A, Evans LE, Alhazzani W, Levy MM, Antonelli M, Ferrer R, et al. Surviving sepsis campaign: International Guidelines for Management of Sepsis and Septic Shock: 2016. Intensive Care Med. 2017;43: 304-377.

9. Atkins E, Bodel P. Clinical fever: its history, manifestations and pathogenesis. Fed Proc. 1979:38:57-63.

10. Peres-Bota D, Lopes-Ferreira F, Mélot C, Vincent JL. Body temperature alterations in the critically ill. Intensive Care Med. 2004;30:811-816.

11. Chiumello D, Gotti M, Vergani G. Paracetamol in fever in critically ill patients-an update. J Crit Care. 2017;38:245-252.

12. Vane JR, Botting RM. Mechanism of action of nonsteroidal anti-inflammatory drugs. Am J Med. 1998:104:2S-8S.

13. Ray JJ, Schulman Cl.J. Fever: suppress or let it ride? J Thorac Dis. 2015;7:E633-E636.

14. Knaus WA, Draper EA, Wagner DP, Zimmerman JE. APACHE II: a severity of disease classification system. Crit Care Med. 1985;13:818-829.

15. Laupland KB, Shahpori R, Kirkpatrick AW, Ross T, Gregson DB, Stelfox HT. Occurrence and outcome of fever in critically ill adults. Crit Care Med. 2008;36:1531-1535.

16. Young P, Saxena M, Eastwood GM, Bellomo R, Beasley R. Fever and fever management among intensive care patients with known or suspected infection: a multicentre prospective cohort study. Crit Care Resusc. 2011;13:97-102.

17. Dinglas VD, Faraone LN, Needham DM. Understanding patient-important outcomes after critical illness: a synthesis of recent qualitative, empirical, and consensus-related studies. Curr Opin Crit Care. 2018:24:401-409.

18. Kushimoto S, Gando S, Saitoh D, Mayumi T, Ogura H, Fujishima S, et al. The impact of body temperature abnormalities on the disease severity and outcome in patients with severe sepsis: an analysis from a multicenter, prospective survey of severe sepsis. Crit Care. 2013;17:R271.

19. Fujishima S, Gando S, Daizoh S, Kushimoto S, Ogura H, Mayumi T, et al. Infection site is predictive of outcome in acute lung injury associated with severe sepsis and septic shock. Respirology. 2016;21:898-904.

20. Paoli CJ, Reynolds MA, Sinha M, Gitlin M, Crouser E. Epidemiology and costs of sepsis in the United States-an analysis based on timing of diagnosis and severity level. Crit Care Med. 2018;46:1889-1897.

21. Young $P$, Saxena M, Beasley R. Acetaminophen for fever in critically ill patients with suspected infection. N Engl J Med. 2016;374:1292-1293.

22. Drewry AM, Ablordeppey EA, Murray ET, Stoll CRT, Izadi SR, Dalton $\mathrm{CM}$, et al. Antipyretic therapy in critically ill septic patients: a systematic review and meta-analysis. Crit Care Med. 2017:45:806-813.

23. Norman DC. Fever in the elderly. Clin Infect Dis. 2000;31:148-151.

24. Fareed A, Scheinberg K, Gale R, Stout S, Vayalapalli, S, Drexler K. Effect of Buprenorphine on liver function tests for patients undergoing long-term maintenance treatment. Addictive Disorders and Their Treatment. 2014;13:133-137. 